\section{Pompe Disease: Presentation and Management of Early Onset Type with Perioperative Considerations}

\section{Abstract}

Early onset Pompe disease is a rare disorder often diagnosed late. Hypotonia, muscle weakness and cardiorespiratory dysfunction are its hallmark. Blood assay of the GAA enzyme is a sensitive and specific marker of the disease. Early intervention with enzyme replacement therapy (ERT) improves morbidity and long term survival, however therapy is both costly and a lifelong requirement. Gene therapy which is on the horizon appears promising.

Keywords: Glycogen storage disease; (GAA) enzyme defiency; Neuro muscular disorder; Infant and adult forms; Multidisciplinary team management; Future prospects

Received: July 09, 2018; Accepted: September 26, 2018; Published: October 03, 2018

\section{Introduction}

Pompe disease is a neuromuscular disease also known as glycogen storage disease type II which is due to a deficiency of the enzyme acid alpha-glucosidase (GAA). This enzyme converts glycogen into glucose. It acts by causing cleavage at the alpha(1,4)-glycosidic bond. Its absence causes glycogen to accumulate in skeletal muscle, smooth muscle and the heart. Pompe disease is also a lysosomal storage disease that results in cell damage and organ dysfunction. Pompe disease is associated with a pathogenic variation of the GAA gene [1-3]. The GYG1 gene type is another gene associated with muscle weakness and glycogen accumulation in the heart [4]. The onset and progress of the disease can occur from birth to late adulthood. Death results with late diagnosis and poor intervention [1]. Being a rare disease diagnosis is often late. The early onset form is a more severe disease with skeletal muscle weakness and wasting causing mobility related difficulties and respiratory failure. There are different subtypes. Two types: infantile onset and late-onset, is distinguished by the age of its onset and the severity of its symptoms [5]. Few symptoms are present at birth and early signs resemble that of "floppy infants" with muscle weakness, hypotonia and a failure to thrive [1]. Disease incidence varies depending on ethnicity and geography. All forms of the disease have an incidence estimated to be 1:40,000 - with males and females being equally affected [1]. Afro-Americans and Chinese manifest with the infantile form and in the Netherlands the lateonset disease is more common (Figure 1).

\section{Bulbulia BA*, Nazeefah Laher and Raeesa Bulbulia}

Garden City Clinic, Johannesburg, South Africa

\section{*Corresponding author: \\ Bulbulia BA}

Garden City Clinic, Johannesburg, South Africa.

”bash786@absamail.co.za

Tel: +0114860389

Citation: Bulbulia BA, Laher N, Bulbulia R (2018) Pompe Disease: Presentation and Management of Early Onset Type with Perioperative Considerations. J Rare Disord Diagn Ther. Vol.4 No.5:21 
lung maturity in the infant and tocolytics to delay labour with a caesarean section planned for later. Having previously had a miscarriage she was considered a high-risk pregnancy. The infant developed hyper- bilirubinemia 140 (N. 5-21 uмоL/L) post-delivery which responded to ultra-violet therapy. No ventillatory support was needed other than supplementary oxygen in the incubator. At age 5 months the child was hospitalized for a chest infection and diagnosed with bronchiolitis. Treatment was symptomatic and uneventful with full recovery. A few weeks later the infant became tachypnoeic and was wheezing. Clinically was found to be in cardiac failure precipitated by another chest infection. A child pulmonologist was consulted and investigations began in earnest upon viewing the chest radiograph (CXR) (Figure 2A and $2 \mathrm{~B}$ ). An urgent echocardiogram (ECHO) showed an ejection fraction of 23 with biventricular enlargement in keeping with a cardiomyopathy (Table 1).

Due to the rarity of Pompe disease suspicion of the disease was delayed.

Differential diagnosis under consideration were:

1. Myocarditis.

2. Idiopathic hypertrophic cardiomyopathy.

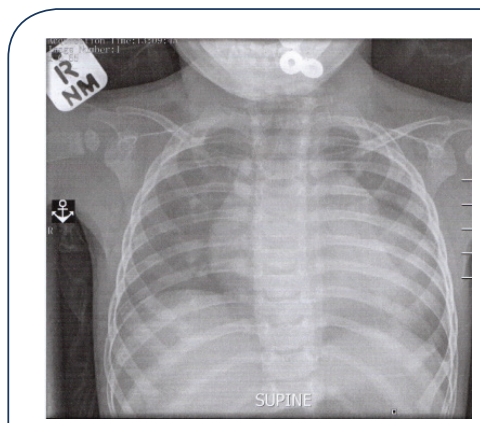

Figure 2A

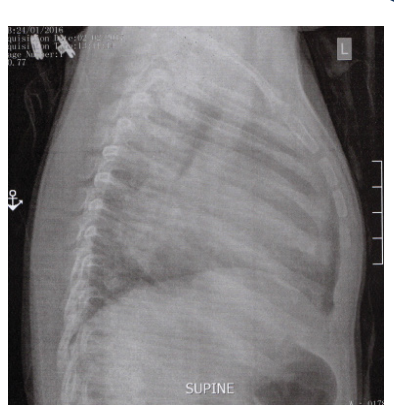

Figure 2B
Figure 2 Chest radiograph showed significant cardiomegaly. Seven month old male with history of Pompe Disease with significant cardiomegaly and a cardiothoracic ratio measuring $67 \%$ and multi-chamber prominence.

Table 1: Laboratory investigations followed.

\begin{tabular}{|c|c|c|}
\hline & Result & Reference \\
\hline $\begin{array}{c}\text { Inflammatory } \\
\text { markers }\end{array}$ & & \\
\hline $\begin{array}{c}\text { C-Reactive Protein } \\
\text { Procalcitonin }\end{array}$ & 1 & $5 \mathrm{mg} / \mathrm{l}$ \\
\hline $\begin{array}{c}\text { Liver Functions } \\
\text { ALT }\end{array}$ & 0.06 & $0.00-0.05 \mathrm{ng} / \mathrm{ml}$ \\
\hline AST & 238 & $<57 \mathrm{u} / \mathrm{l}$ \\
\hline Cardiac Markers & 447 & $<89 \mathrm{u} / \mathrm{l}$ \\
\hline Myoglobin & & $17-10^{6} \mathrm{ng} / \mathrm{ml}$ \\
\hline CK-MB & 257 & $0-7.6$ \\
\hline Tropinin T & 50.1 & $0-14$ \\
\hline NT- proBNP & 61 & $\mathrm{ng} / \mathrm{ml}$ \\
\hline CK (Creatine Kinase) & 31827 & $<125$ \\
\hline Thyroid Functions & 1927 & $\mathrm{normal}$ \\
\hline
\end{tabular}

3. Congenital muscle dystrophic hypotonia.

On the opinion of a second Pead Cardiologist enzyme assays on blood was tested for the GAA enzyme. The level confirmed lower than normal activity levels:

1. Patient activity 2.28 units

2. Normal control 10.89 units

3. Normal range for patient activity $7.96-19.32$ units

This finding was consistent with Pompe disease. Dried blood done for molecular genetic testing confirmed Pompe disease. Two mutations were detected. The c. (1933 G > A); (1933G>A). Genetic profiling of the parents showed both to be carriers of the recessive gene. One mutation was detected in each parent. The heterozygous mutation c. (1933G>A). Enzyme replacement therapy was begun with Myozyme as well as cardiac support therapy instituted which included an Ace- inhibitor and antifailure therapy. To date no adverse reaction has occurred with the infusions or anti-body build up detected. Home care infusions have now replaced in hospital infusions.

A steady improvement in the skeletal myopathy was observed and remodelling of the heart with a reversal of the cardiomyopathy was seen at 2 years. Echocardiogram reported normal cardiac function and ejection fraction (Figure 3).

\section{Discussion}

\section{Infantile onset}

- Classic Infantile Pompe disease: a severe form that is rapidly progressive and causes early death [1].

- Non-classic Infantile Pompe disease: is characterized by slower progression and less severe cardiomyopathy [1].

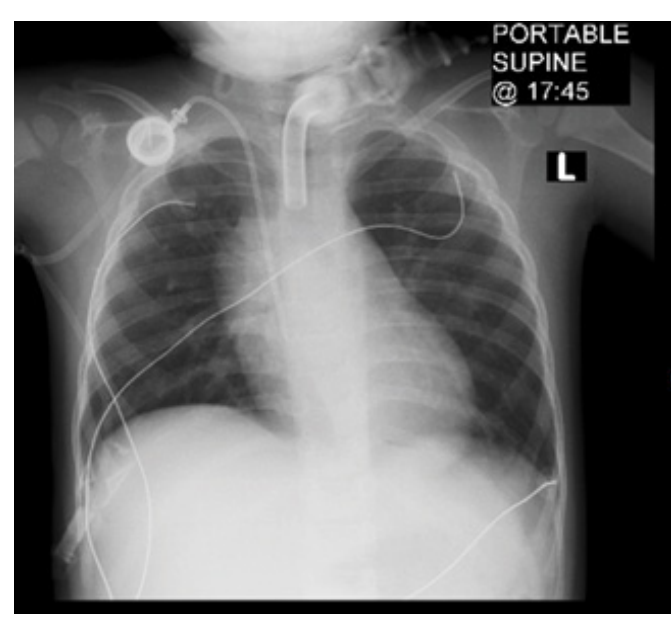

Figure 32 year 2 month old male patient following Myozyme therapy for Pompe disease. Heart is not enlarged and reversal of cardiomyopathy. (Same patient as imaged above at 7 months). Central Line and Traechosty Tube in situ. Following failed extubation attempts after anaesthesia for the insertion of feeding gastro-enterosty tube (PEG) and the insertion of a central line a trachyostomy tube was inserted. Patient was discharged on home ventilation and oxygen. 
Presenting features are cardiac enlargement, feeding difficulties and skeletal muscle weakness. Untreated life expectancy is short.

\section{Late-onset}

Have mobility related difficulties and respiratory weakness with fewer cardiac problems. Disease may occur during childhood, adolescence or adulthood. There are distinct patterns between infantile onset and late-onset disease [1]. Kishnani and colleagues state that: "Phenotypic variability has led to the creation of types based on the age of onset and degree of organ involvement. Pompe disease is a continuum of disease spectrum varying by age of onset, organ involvement and degree of myopathy" [2]. The signs \& symptoms illustrate a typical case of classic infantile Pompe disease. Cardio-respiratory dysfunction occurred early (within its first year of life), feeding and swallowing difficulties and a large protruding tongue. In infantile onset physical developmental milestones delays such as sitting up, standing and walking occur [1]. Mental development and cognitive function remain unimpaired and the child has shown remarkable learning and adaptive skills. He has the ability to mimic sounds, recognize pictures and faces and communicate needs and explore his environment. For late onset Pompe disease, developmental milestones are normal and presentation delayed. Both groups are associated with progressive muscle weakness which is highly variable [1]. Proximal muscles strength and respiratory muscles are affected. Lower limbs weakness may be more pronounced and those supporting the axial skeleton and spine impacted. Pelvic girdle weakness is more obvious than upper limbs and shoulders. Scoliosis and other skeletal abnormalities can occur. At 2 y 9 months the patient is able to sit unaided and has good shoulder and upper limb muscle tone and power. Lower limb strength is poor and movement against gravity difficult. Mobility aids and wheelchair assist devices may be required for the future. Respiratory assistance and ventilator support is being used and home ventilation given with continuous positive airway pressure (CPAP).

\section{Diagnosis and investigations}

Clinical evaluation is based on muscular, respiratory, and/or cardiac history and examination [1,2]. The investigations and laboratory findings were suggestive of a neuromuscular disorder and severe cardiac decompensation. Other conditions that needed consideration were;

\section{- Carnitine deficiency}

- Danons disorder

\section{- Congenital muscle dystrophy}

They share in common some features of skeletal myopathy, weakness, cardiomyopathy and elevated muscle enzymes. The low levels of the GAA enzyme and the subsequent genetic profiling confirmed the diagnosis of Pompe disease in this case.

\section{Routine tests to include}

- Chest x-ray and electrocardiogram (ECG).

- Echocardiogram.

- A chest $\mathrm{x}$-ray will show cardiomegaly. In late onset cases cardiomegaly is not often seen.

- Blood tests for serum creatine kinase (CK), a sensitive but non- specific marker. CK levels may be in the normal range. Normal or may be elevated in infantile - onset patients [1].

- Lung function test will identify at risk patients but is not practical in little infants.

- Liver function tests may be useful in testing for both infantile and late-onset Pompe disease. Elevated transaminases may occur as well derangements of other enzymes.

\section{- Cardiac enzymes}

Confirmed diagnosis is made on a blood spot assay or on laboratory blood testing of the GAA enzyme level along with molecular genetic confirmation which remains the gold standard [6]. Pre-natally fibroblasts taken from amniotic is helpful for genetic diagnosis.

\section{Treatment, Management and Therapy}

The treatment and management of Pompe remains disease specific and affects multiple organ systems. A multidisciplinary management team includes: neurologists, cardiologists, pulmonologists, gastroenterologists, dieticians, physiotherapists, speech therapists, occupational therapists, and other healthcare professionals [1]. Affected individuals and families will need genetic counselling and advice on future family planning [1].

\section{Enzyme Replacement Therapy (ERT)}

This is the only treatment available for Pompe disease. It involves the intravenous administration of recombinant human acid $\alpha$-glucosidase, known as Myozyme for all patients with the disease. ERT has been shown to improve muscle and cardiac symptoms and longevity. Infusions are given weekly or forthnightly with a standard dose of $20 \mathrm{mg} / \mathrm{kg}$. There may be added benefits at $40 \mathrm{mg} / \mathrm{kg}$ in classic infantile forms of the disease. The GAA protein has a short half-life and is safe but anaphylaxis may occur. Monitoring for IgG antibodies must be done regularly. Home infusions are a reality and with proper training and precautions are being done. ERT is lifelong and costly. In dollar terms may be $\$ 10,000 /$ month upwards. This has huge implications for cost containment and health care funders and local government may resist such funding.

\section{Supportive therapies}

Many patients experience respiratory difficulties. Respiratory support includes mechanical ventilation, Bi-level Positive Airway Pressure (Bipap) or Continuous Positive Airway Pressure (CPAP). The treatment period is patient specific. Additional timely intervention for respiratory infections and pulmonary toilet measures are important.

Physiotherapy and Occupational Therapy are needed to develop and maintain patient strength and physical ability. Mobility or supporting aids may be necessary along with splinting to prevent deformities or contractures. Speech therapy and feeding therapy are essential for the development and maintenance of oral motor skills for speech, swallowing and feeding. Dietary planning, 
nutritional status and adequate weight gain is essential. Patients may require high-calorie diets and how to manage feeds, change food texture in order to lower the risk of gastric regurgitation and pulmonary aspiration. The placement of a feeding gastoenterostomy tube (PEG) may be required.

\section{Gene therapy}

Gene therapy appears promising and remains an exciting option [7-9]. This is aimed at restoring acid alpha glocosidase production and activity by transducing the functional GAA gene into liver cells [9].

\section{Perioperative considerations}

General anaesthesia will be a requirement for:

1. Insertion of a central line and port to facilitate enzyme infusions.

2. [PEG] Gastro-enterostomy tube insertion for feeds.

3. Tracheostomy

4. Release of muscle contractures

\section{References}

1. National Organization for Rare Disorders. Pompe Disease (2018) https://rarediseases.org/rare-diseases/pompe-disease/

2. Kishnani PS, Steiner RD, Bali D, Berger K, Byrne BJ, et al. (2006) Pompe disease diagnosis and management guideline. Genet Med 8 : 267.

3. Hers HG (1963) $\alpha$-Glucosidase deficiency in generalized glycogenstorage disease (Pompe's disease). Biochem J 86: 11.

4. https://themedicalbiochemistrypage.org/glycogen.php

5. Kishnani PS, Howell RR (2004) Pompe disease in infants and children. J Pediatr 144: S35-S43.

6. Hirschhorm R (2001) Glycogen storage disease type II. Acid alphaglucosidase (acid maltase) deficiency. The metabolic and molecular bases of inherited disease. Pp: 3389-3420.

7. Kishnani PS, Howell RR (2004) Pompe disease in infants and children. J Pediatr 144: S35-S43.
Anaesthesia for children remains challenging. Intubation difficulties due to mouth size and a large tongue may occur. Cardio-pulmonary dysfunction may be present. Chronic medication prescribed requires good communication between anaesthesiologist and other role players. With a background of Cardiomyopathy, a cardiology consultation is essential. The use of muscle relaxants should be titrated or reduced and reversed to ensure full recovery.

\section{Conclusion}

With advances in medical technology remarkable progress is in the making. Newer therapies in genetics and molecular biology promise a better outlook and will have a major impact in morbidity and mortality in the near future. Human clinical trials are only starting with the development of an adeno-associated virus vector to transduce the GAA gene into the liver and have a continuous supply of the GAA protein. Uptake of the enzyme occurs in heart and skeletal muscle and glycogen cleared. Only a single administration is required [9]. Pompe disease children have special needs that call for patience, dedication, and support from multiple role players [8-12].

8. Hagemans MLC, Winkel LPF, Van Doorn PA, Hop WJC, Loonen MCB, et al. (2005). Clinical manifestation and natural course of late-onset Pompe's disease in 54 Dutch patients. Brain 128: 671-677.

9. National Center for Advancing Translational Sciences. Gene Therapy for the Treatment of Pompe Disease (2018) https://ncats.nih.gov/ trnd/projects/active/treatment-pompe-disease.

10. Ausems MGEM, Verbiest J, Hermans MMP, Kroos MA, Beemer FA, et al. (1999) Frequency of glycogen storage disease type II in The Netherlands: implications for diagnosis and genetic counselling. Eur J Hum Genet 7: 713.

11. Byrne BJ, Kishnani PS, Case LE, Merlini L, Müller-Felber W, et al. (2011) Pompe disease: design, methodology, and early findings from the Pompe Registry. Mol Gen Metab 103: 1-11.

12. Hagemans MLC, Winkel LPF, Van Doorn PA, Hop WJC, Loonen MCB, et al. (2005) Clinical manifestation and natural course of late-onset Pompe's disease in 54 Dutch patients. Brain 128: 671-677. 